\title{
Fixed point results in non-Archimedean generalized intuitionistic fuzzy metric spaces
}

\author{
M. Jeyaraman ${ }^{1}$, M. Suganthi ${ }^{2}$ and S. Sowndrarajan ${ }^{3}$ \\ ${ }^{1}$ PG and Research Department of Mathematics, \\ Raja Doraisingam Govt. Arts College, Sivagangai, India \\ Affiliated Alagappa University, Karaikudi, India \\ e-mail: jeya.mathegmail.com \\ ${ }^{2}$ Research Scholar, Department of Mathematics, Govt. Arts College, Melur \\ PG and Research Department of Mathematics, \\ Raja Doraisingam Govt. Arts College, Sivagangai, \\ Affiliated Alagappa University, Karaikudi, India \\ e-mail: vimalsugan@gmail.com \\ ${ }^{3}$ Research Scholar, PG and Research Department of Mathematics, \\ Raja Doraisingam Govt. Arts College, Sivagangai, \\ Affiliated Alagappa University, Karaikudi, India \\ e-mail: sowndariitmegmail.com
}

\begin{abstract}
In this paper, we introduce the concept of non-Archimedean generalized intuitionistic fuzzy metric space and obtain some results for two semi compatible mappings in this newly defined space. Our results improve and generalize the results of Mustafa et al. [8] and Abbas and Rhoades [1] in non-Archimedean $G$-fuzzy metric space.
\end{abstract}

Keywords: $G$-metric space, Non-archimedean, Fuzzy metric space, Generalized fuzzy metric space, Common fixed point.

2010 Mathematics Subject Classification: 47H10, 46B20.

\section{Introduction}

In mathematics, the concept of fuzzy sets was introduced by Zadeh [13]. It is a new way to represent vagueness in our daily life. In 1975, Kramosil and Michalek [6] introduced the concept 
of fuzzy metric spaces which opened a new way for further development of analysis in such spaces. George and Veeramani [4] modified the concept of fuzzy metric space. After that, several fixed point theorems proved in fuzzy metric spaces. In 2006, Mustafa and Sims [7] presented a definition of $G$-metric space. After that, several fixed point results proved in $G$-metric spaces. On the other hand, Atanassov [2] introduced the concept of intuitionistic fuzzy sets as a generalization of fuzzy sets. Park [9] has introduced and studied the notion of intuitionistic fuzzy metric spaces. Further, Saadati et al. [11] proposed the idea of a continuous $t$ representable under the name modified intuitionistic fuzzy metric space which is a milestone in developing fixed point theory.

Motivated by the concepts of $G$-metric space, non-Archimedean metric space and Fuzzy metric space, we introduce the concept of non-Archimedean generalized intuitionistic fuzzy metric space and obtain two common fixed point theorems for two semi compatible mappings. In this paper, we introduce the notion of generalized intuitionistic fuzzy metric space and describe some of their properties of generalized fuzzy metric space. Our results improve and generalize the results of Mustafa et. al. [8] and Abbas and Rhoades [1] in non-Archimedean $G$-fuzzy metric space. We also establish properties $P$ and $Q$ for these mappings.

\section{Preliminaries}

Definition 2.1. A 5-tuple $\left(X, G, H,{ }^{*}, \diamond\right)$ is said to be a generalized intuitionistic fuzzy metric space, if $X$ is an arbitrary non-empty set, * is a continuous $t$-norm, $\diamond$ is a continuous $t$-conorm, $G$ and $H$ are fuzzy sets on $X^{3} \times(0, \infty)$ satisfying the following conditions: for every $x, y, z, a$ $\in X$ and $t, s>0$.

(i) $\quad G(x, y, z, t)+H(x, y, z, t) \leq 1$,

(ii) $\quad G(x, x, y, t)>0$ for $x \neq y$,

(iii) $\quad G(x, x, y, t) \geq G(x, y, z, t)$ for $y \neq z$,

(iv) $\quad G(x, y, z, t)=1$ if and only if $x=y=z$,

(v) $G(x, y, z, t)=G(p(x, y, z), t)$, where $p$ is a permutation function,

(vi) $G(x, a, a, t) * G(a, y, z, s) \leq G(x, y, z, t+s)$,

(vii) $G(x, y, z,):.(0, \infty) \rightarrow[0,1]$ is continuous.

(viii) $G$ is a non-decreasing of $\mathbb{R}^{+}, \lim _{\mathrm{t} \rightarrow \infty} G(x, y, z, t)=1, \lim _{t \rightarrow 0} G(x, y, z, t)=0$ for all $x, y, z \in X$ and $t>0$

(ix) $\quad H(x, x, y, t)<1$ for $x \neq y$,

(x) $\quad H(x, x, y, t) \leq H(x, y, z, t)$ for $y \neq z$,

(xi) $\quad H(x, y, z, t)=0$ if and only if $x=y=z$,

(xii) $H(x, y, z, t)=H(p(x, y, z), t)$, where $p$ is a permutation function,

(xiii) $H(x, a, a, t) \diamond H(a, y, z, s) \geq H(x, y, z, t+s)$,

(xiv) $H(x, y, z,):.(0, \infty) \rightarrow[0,1]$ is continuous.

(xv) $\quad G$ is a non-increasing of $\mathbb{R}^{+}, \lim _{t \rightarrow \infty} H(x, y, z, t)=0, \lim _{t \rightarrow 0} H(x, y, z, t)=1$ for all $x, y, z \in X$ and $t>0$.

In this case, the pair $(G, H)$ is called a generalized intuitionistic fuzzy metric on $X$. 
Definition 2.2. A 5-tuple $(X, G, H, *, \diamond)$ is said to be a non-Archimedean generalized intuitionistic fuzzy metric space (shortly GIFM-Space), if $X$ is an arbitrary set, * is a continuous $t$-norm, $\diamond$ is a continuous $t$-conorm and $G$ and $H$ are fuzzy sets on $X^{3} \times(0, \infty)$ satisfying the following conditions: for all $x, y, z, a \in X$ and $s, t>0$.

(i) $\quad$ (GIFM 1) $G(x, y, z, t)+H(x, y, z, t) \leq 1$,

(ii) (GIFM 2) $G(x, y, z, t)>0$,

(iii) (GIFM 3) $G(x, y, z, t)=1$ if and only if $x=y=z$,

(iv) (GIFM 4) $G(x, y, z, t)=G(p\{x, y, z\}, t)$, where $p$ is a permutation function,

(v) (GIFM 5) $G(x, y, z, \max \{t, s\}) \geq G(x, y, a, t) * G(a, z, z, s)$,

(vi) (GIFM 6) $G(x, y, z,):.(0, \infty) \rightarrow[0,1]$ is continuous,

(vii) (GIFM 7) $H(x, y, z, t)>0$,

(viii) (GIFM 8) $H(x, y, z, t)=0$ if and only if $x=y=z$,

(ix) (GIFM 9) $H(x, y, z, t)=H(p\{x, y, z\}, t)$, where $p$ is a permutation function,

(x) $\quad($ GIFM 10) $H(x, y, z, \min \{t, s\}) \leq H(x, y, a, t) \diamond H(a, z, z, s)$,

(xi) $\quad($ GIFM 11) $H(x, y, z,):.(0, \infty) \rightarrow[0,1]$ is continuous.

Then, $(G, H)$ is called a generalized intuitionistic fuzzy metric space on $X$.

The functions $G(x, y, z, t)$ and $H(x, y, z, t)$ denote the degree of nearness and degree of nonnearness between $x, y$ and $z$ with respect to $t$, respectively.

Remark 2.3. In generalized intuitionistic fuzzy metric space $X . G(x, y, z,$.$) is non-decreasing$ and $H(x, y, z,$.$) is non-increasing for all x, y, z \in X$.

In the above definition, if the triangular inequality (GIFM 5) and (GIFM 10) are replaced by the following:

$$
\begin{aligned}
& G(x, y, z, \max \{t, s\}) \geq G(x, y, a, t) * G(a, z, z, s) \\
& H(x, y, z, \min \{t, s\}) \leq H(x, y, a, t) \diamond H(a, z, z, s),
\end{aligned}
$$

or equivalently

$$
\begin{aligned}
& G(x, y, z, t) \geq G(x, y, a, t) * G(a, z, z, t) \\
& H(x, y, z, t) \leq H(x, y, a, t) \diamond H(a, z, z, t) .
\end{aligned}
$$

Then $(X, G, H, *, \diamond)$ is called non-Archimedean generalized intuitionistic fuzzy metric space. It is easy to check that the triangle inequality (NA) implies (GIFM 5) and (GIFM 10), that is, every non-Archimedean generalized intuitionistic fuzzy metric space is itself a generalized intuitionistic fuzzy metric spaces.

Definition 2.4. Denote by $\Phi$ the class of continuous functions $\phi, \psi:[0,1] \rightarrow[0,1]$ such that $\phi(t)>t$ for all $0 \leq t<1$ and $\phi(1)=1$ and $\psi(t)<t$ for all $0 \leq t<1$ and $\psi(0)=0$.

Lemma 2.5. Let $(X, \mathrm{G}, \mathrm{H}, *, \diamond)$ be a non-Archimedean generalized intuitionistic fuzzy metric space. Than $G(x, y, z,$.$) is non-decreasing and H(x, y, z,$.$) is non-increasing with respect to t$ for all $x, y, z$ in $X$.

Throughout this paper, we assume that $\lim _{n \rightarrow \infty} G(x, y, z, t)=1$ and $\lim _{n \rightarrow \infty} H(x, y, z, t)=0$ and that $N$ is the set of all natural numbers. 
Lemma 2.6. Let $\left(X, G, H,{ }^{*}, \diamond\right)$ be a non-Archimedean generalized intuitionistic fuzzy metric space. Let $\left\{y_{n}\right\}$ be a sequence in $X$, where $*$ is a continuous $t$-norm, $\diamond$ is a continuous $t$-conorm satisfying $t * t \geq t$ and $1-t \diamond 1-t \leq 1-t$, for all $t \in[0,1]$. If there exists $t>0$ and $\phi \in \Phi$ and $\psi \in \Psi$ such that $G\left(y_{n+1}, y_{n+2}, y_{n+2}, t\right) \geq \phi\left(G\left(y_{n}, y_{n+1}, y_{n+1}, t\right)\right)$ and $H\left(y_{n+1}, y_{n+2}, y_{n+2}, t\right)$ $\leq \psi\left(H\left(y_{n}, y_{n+1}, y_{n+1}, t\right)\right), n \in N$, then $\left\{y_{n}\right\}$ is a Cauchy sequence in $X$.

Proof. If we define $r_{n}=G\left(y_{n+1}, y_{n+2}, y_{n+2}, t\right)$ and $p_{n}=H\left(y_{n+1}, y_{n+2}, y_{n+2}, t\right)$, then

$$
r_{n} \geq \phi\left(r_{n-1}\right)>r_{n-1} \text { and } p_{n} \leq \psi\left(p_{n-1}\right)<p_{n-1}
$$

So, that the sequence $\left\{r_{n}\right\}$ is an increasing sequence of positive real numbers in $[0,1]$ and tends to a limit $r \leq 1$. We claim that $r=1$. If $r<1$, on taking $n \rightarrow \infty$ in (2.6.1), we get $r \geq \varnothing(r)>r$, which is a contradiction. Hence $r=1$.

So, that the sequence $\left\{p_{n}\right\}$ is a decreasing sequence of positive real numbers in $[0,1]$ and tends to a limit $p \geq 0$. We claim that $p=0$. If $p>1$, on taking $n \rightarrow \infty$ in (2.6.1), we get $p \leq \psi(p)<p$, which is a contradiction. Hence $p=0$.

Now, for any positive integer $p$, we have

$$
\begin{aligned}
& G\left(y_{n}, y_{n+p}, y_{n+p}, t\right) \geq G\left(y_{n}, y_{n+1}, y_{n+1}, t\right) * \ldots * G\left(y_{n+p-1}, y_{n+p}, y_{n+p}, t\right) . \\
& H\left(y_{n}, y_{n+p}, y_{n+p}, t\right) \leq H\left(y_{n}, y_{n+1}, y_{n+1}, t\right) \diamond \ldots \diamond H\left(y_{n+p-1}, y_{n+p}, y_{n+p}, t\right) .
\end{aligned}
$$

Taking the limit as $n \rightarrow \infty$, we get $\lim _{n \rightarrow \infty} G\left(y_{n}, y_{n+p}, y_{n+p}, t\right)=1$ and $\lim _{n \rightarrow \infty} H\left(y_{n}, y_{n+p}, y_{n+p}, t\right)$ $=0$. Hence, $\left\{y_{n}\right\}$ is a Cauchy sequence.

Now, we introduce the concept of weekly compatible maps and semi-compatible maps in non-Archimedean generalized intuitionistic fuzzy metric space as follows.

Definition 2.7. Let $f$ and $g$ be self-maps on a non-Archimedean generalized intuitionistic fuzzy metric space $(X, G, H, *, \diamond)$. Then the mappings are said to be weakly compatible if they commute at their coincidence point, that is, $f x=g x$ implies that $f g h=g f h$.

Definition 2.8. A pair $(f, g)$ of self-mappings of a non-Archimedean generalized intuitionistic Fuzzy metric space is said to be semi-compatible if $\lim _{n \rightarrow \infty} f g x_{n}=g x$, whenever $\left\{x_{n}\right\}$ is a sequence in $X$ such that $\lim _{n \rightarrow \infty} f x_{n}=\lim _{n \rightarrow \infty} g x_{n}=x$, for some $x \in X$.

It follows that $(f, g)$ is semi-compatible and $f y=g y$, then $f g y=g f y$.

Note that every pair of semi-compatible maps are weakly compatible, but the converse need not be true.

Example 2.9. Let $X=[0,1]$ with $G$-metric on $X$ defined by $G(x, y, z)=|x-y|+|y-z|+|z-x|$. Denote $a * b=a b$ and $a \diamond b=\min \{1, a+b\}$ for all $a, b \in[0,1]$. For all $x, y, z \in X$ and $t>0$, define $G$ on $X^{3} \times(0, \infty)$ as follows:

$$
\mathrm{G}(x, y, z, t)=\left(\frac{t}{t+1}\right)^{G(x, y, z)} \quad \text { and } \quad H(x, y, z, t)=\left(\frac{1}{t+1}\right)^{H(x, y, z)} .
$$

Then, $(X, G, H, *, \diamond)$ is a non-Archimedean generalized intuitionistic fuzzy metric space. Define a self-map on $X$ as follows:

$$
S x=\left\{\begin{array}{c}
x 0 \leq x<\frac{1}{2} \\
1 x \geq \frac{1}{2}
\end{array}\right\} \text { and let } I \text { be the identity map on } X .
$$


Definition 2.10. Let $f$ and $g$ be self-maps on a set $X$ and if $w=f x=g x$ for some $x$ in $X$, then $x$ is called a coincidence point of $f$ and $g$ and $\mathrm{w}$ is called a point of coincidence of $f$ and $g$.

Preposition 2.11. Let $f$ and $g$ be semi-compatible self-maps of a set $X$. If $f$ and $g$ have a unique point of coincidence $f x=g x=w$, then $\mathrm{w}$ is the unique common fixed point of $f$ and $g$.

Proof. Since $f x=g x=w$ and $f$ and $g$ are semi-compatible, we have $f w=f g h=g h f=g w$, implies that $f w=g w$. Thus, $w$ is a point of coincidence of $f$ and $g$. But $w$ is the only point of coincidence of $f$ and $g$, so $w=f w=g w$. Moreover, if $z=f z=g z$, then $z$ is a point of coincidence of $f$ and $g$. Therefore $z=w$, by uniqueness. Thus, $w$ is the unique common fixed point of $f$ and g.

Definition 2.12. Let $\left(X, G, H,{ }^{*}, \diamond\right)$ be a non-Archimedean generalized intuitionistic fuzzy metric space and $T: X \rightarrow X$ be a mapping with fixed point set $F(T) \neq \varnothing$. Then $T$ has property $P$ if $F\left(T^{n}\right)$ $=F(T)$, for each $n \in N$.

Definition 2.13. Let $\left(X, G, H,{ }^{*}, \diamond\right)$ be a non-Archimedean generalized intuitionistic fuzzy metric space and $T, S: X \rightarrow X$ be two mappings with $F(S) \cap F(T) \neq \varnothing$. Then $T$ and $S$ have property $Q$ if $F\left(S^{n}\right) \cap F\left(T^{n}\right)=F(S) \cap F(T)$, for each $n \in N$.

\section{Main results}

Now, we generalize the results of Abbas and Rhoades [1] to non-Archimedean generalized intuitionistic fuzzy metric space for semi-compatible maps as follows:

Theorem 3.1. Let $\left(X, G, H,{ }^{*}, \diamond\right)$ be a non-Archimedean generalized intuitionistic fuzzy metric space with $t \geq t$ and $(1-t) \diamond(1-t) \leq(1-t)$. Suppose $f$ and $g$ be a self-map of $X$ satisfying for all $x, y, z \in X$,

$$
\begin{aligned}
& G(f x, f y, f z, t) \geq \phi(G(g x, g y, g z, t)), \\
& H(f x, f y, f z, t) \leq \psi(H(g x, g y, g z, t)),
\end{aligned}
$$

where $\phi \in \Phi, \psi \in \Psi, t>0$. If $f(X) \subset g(X)$ and $g(X)$ is a complete subspace of $X$, then $f$ and $g$ have a unique point of coincidence in $X$. Moreover, if $f$ and $g$ are semi-compatible, then $f$ and $g$ have a unique common fixed point.

Proof. Let $x_{0}$ be a point in $X$. Since $f(X) \subset g(X)$, so we choose a point $x_{1}$ in $X$ such that $f\left(x_{0}\right)=g\left(x_{1}\right)$. Continuing this process, having chosen $x_{n}$ in $X$, we can find $x_{n+1}$ in $X$ such that

$$
y_{n}=f x_{n}=g x_{n+1}, n=0,1,2, \ldots
$$

Now, we prove that $\left\{y_{n}\right\}$ is a Cauchy sequence. Then, by (3.1.1), we have

$$
\begin{aligned}
G\left(y_{n}, y_{n+1}, y_{n+1}, t\right) & =G\left(f x_{n}, f x_{n+1}, f x_{n+1}, t\right) \\
& \geq \phi\left(G\left(g x_{n}, g x_{n+1}, g x_{n+1}, t\right)\right)=\phi\left(G\left(y_{n-1}, y_{n}, y_{n}, t\right) .\right. \\
H\left(y_{n}, y_{n+1}, y_{n+1}, t\right) & =H\left(f x_{n}, f x_{n+1}, f x_{n+1}, t\right) \\
& \leq \psi\left(H\left(g x_{n}, g x_{n+1}, g x_{n+1}, t\right)\right)=\psi\left(H\left(y_{n-1}, y_{n}, y_{n}, t\right) .\right.
\end{aligned}
$$


Then, Lemma (2.6), $\left\{y_{n}\right\}$ is a Cauchy sequence. This implies that $\left\{g x_{n}\right\}$ is a Cauchy sequence. Since $g(X)$ is complete, so there exists $u \in g(X)$ such that

$$
\lim _{n \rightarrow \infty} y_{n}=\lim _{n \rightarrow \infty} f x_{n}=\lim _{n \rightarrow \infty} g x_{n}=u \text {. }
$$

Since $u \in g(X)$, so there exists $p \in X$ such that $g p=u$. Let $f p \neq u$. From (3.1.1)

$$
G\left(f x_{n}, f p, f p, t\right) \geq \phi\left(G\left(g x_{n}, g p, g p, t\right)\right)
$$

as $n \rightarrow \infty$, we get

$$
G(u, f p, f p, t) \geq \phi(G(g p, g p, g p, t))=\phi(1)=1 .
$$

This implies that $G(u, f p, f p, t)=1, H\left(f x_{n}, f p, f p, t\right) \leq \psi\left(H\left(g x_{n}, g p, g p, t\right)\right)$, as $n \rightarrow \infty$, we get

$$
H(u, f p, f p, t) \leq \psi(H(g p, g p, g p, t))=\psi(0)=0 .
$$

This implies that $H(u, f p, f p, t)=0$, which is a contradiction, since $f p \neq u$.

Thus, $f p=g p=u$. Hence, $\mathrm{p}$ is a coincidence point of $f$ and $g$.

Now, we will show that $p$ is unique. Assume that there exists another point $q$ in $X$ such that $f q=g q$. If $f p \neq f q$, then

$G(f q, f p, f p, t) \geq \phi(G(g q, g p, g p, t))=\phi(G(f q, f p, f p, t))>G(f q, f p, f p, t)$,

$H(f q, f p, f p, t) \leq \psi(H(g q, g p, g p, t))=\psi(H(f q, f p, f p, t))<H(f q, f p, f p, t)$,

which is a contradiction. Hence $f p=f q$.

Moreover, if $f$ and $g$ are semi-compatible, then from proposition (2.11), $f$ and $g$ have a unique common fixed point.

If we take $g=1$ in Theorem 3.1, we obtain the following result:

Corollary 3.2. Let $(X, \mathrm{G}, \mathrm{H}, *, \diamond)$ be a non-Archimedean generalized intuitionistic fuzzy metric space with $t^{*} t \geq \mathrm{t}$ and $(1-t) \diamond(1-t) \leq(1-t)$. Suppose $f$ is a self-map of $X$ satisfying for all $x, y, z \in X$

$$
G(f x, f y, f z, t) \geq \phi(G(x, y, z, t))
$$

and

$$
H(f x, f y, f z, t) \leq \psi(H(x, y, z, t)),
$$

where $t>0$ and $\phi \in \Phi$ and $\in \Psi$. Then $f$ has a unique fixed point.

Theorem 3.3. Let $(X, \mathrm{G}, \mathrm{H}, *, \diamond)$ be a non-Archimedean generalized intuitionistic fuzzy metric space with $t^{*} t \geq \mathrm{t}$ and $(1-t) \diamond(1-t) \leq(1-t)$. If the mappings $f, g: X \rightarrow X$ satisfy either

and

$$
G(f x, f y, f z, t) \geq \phi(\min \{G(g x, f x, f x, t), G(g y, g y, f y, t), G(g z, f z, f z, t)\})
$$

$$
H(f x, f y, f z, t) \leq \psi(\max \{H(g x, f x, f x, t), H(g y, g y, f y, t), H(g z, f z, f z, t)\})
$$

or

and

$$
G(f x, f y, f z, t) \geq \phi(\min \{G(g x, g x, f x, t), G(g y, g y, f y, t), G(g z, g z, f z, t)\})
$$

$H(f x, f y, f z, t) \leq \psi(\max \{H(g x, g x, f x, t), H(g y, g y, f y, t), H(g z, g z, f z, t)\})$ 
for all $x, y, z \in X$ where $\phi \in \Phi$ and $\psi \in \Psi, t>0$. If $f(X) \subset g(X)$ and $g(x)$ is a complete subspace of $X$ than $f$ and $g$ have a unique point of coincidence in $X$. Moreover, if $f$ and $g$ are semicompatible, then $f$ and $g$ have a unique common fixed point.

Proof. Suppose that $f$ and $g$ satisfy (3.3.1). Let $x_{0}$ be an arbitrary point in $X$. Since $f(X) \subset g(X)$, so we choose a point $x_{1}$ in $X$ such that $f\left(x_{0}\right)=g\left(x_{1}\right)$. Continuing this process, having chosen $x_{n}$ in $X$, we can find $x_{n+1}$ in $X$ such that $f\left(x_{n}\right)=g\left(x_{n+1}\right)$. Inductively, construct a sequence $\left\{y_{n}\right\}$ in $X$ such that

$$
y_{n}=f x_{n}=g x_{n+1}, n=0,1 \ldots
$$

Now, we prove that $\left\{y_{n}\right\}$ is a Cauchy sequence. Then, by (3.3.1), we have

$$
\begin{aligned}
G\left(y_{n}, y_{n+1}, y_{n+1}, t\right) & =G\left(f x_{n}, f x_{n+1}, f x_{n+1}, 1\right) \\
\geq & \phi\left(\min \left\{G\left(g x_{n}, f x_{n}, f x_{n}, t\right), G\left(g x_{n+1}, f x_{n+1}, f x_{n+1}, t\right), G\left(g x_{n+1}, f x_{n+1}, f x_{n+1}, t\right)\right\}\right) \\
& \left.=\phi\left(\min G\left(y_{n-1}, y_{n}, y_{n}, t\right), G\left(y_{n}, y_{n+1}, y_{n+1}, t\right), G\left(y_{n}, y_{n+1}, y_{n+1}, t\right)\right\}\right) \\
H\left(y_{n}, y_{n+1}, y_{n+1}, t\right) & =H\left(f x_{n}, f x_{n+1}, f x_{n+1}, 1\right) \\
& \leq \psi\left(\max \left\{H\left(g x_{n}, f x_{n}, f x_{n}, t\right), H\left(g x_{n+1}, f x_{n+1}, f x_{n+1}, t\right), H\left(g x_{n+1}, f x_{n+1}, f x_{n+1}, t\right)\right\}\right) \\
& \left.=\psi\left(\max H\left(y_{n-1}, y_{n}, y_{n}, t\right), H\left(y_{n}, y_{n+1}, y_{n+1}, t\right), H\left(y_{n}, y_{n+1}, y_{n+1}, t\right)\right\}\right) .
\end{aligned}
$$

Thus, we obtain

$$
\begin{aligned}
& G\left(y_{n}, y_{n+1}, y_{n+1}, t\right) \geq \phi\left(\min \left\{G\left(y_{n-1}, y_{n}, y_{n}, t\right), G\left(y_{n}, y_{n+1}, y_{n+1}, t\right)\right\}\right), \\
& H\left(y_{n}, y_{n+1}, y_{n+1}, t\right) \leq \psi\left(\max \left\{H\left(y_{n-1}, y_{n}, y_{n}, t\right), H\left(y_{n}, y_{n+1}, y_{n+1}, t\right)\right\}\right) .
\end{aligned}
$$

Without loss of generality, assume $y_{n} \neq y_{n+1}$ for each $n$. (Since, if there exists an $n$ such that $y_{n}=y_{n+1}$, then $y_{n}=f x_{n}=g x_{n+1}=f x_{n+1}=g x_{n+2}$, implies that, $g x_{n+1}=f x_{n+1}$. Then, $f$ and $g$ have a coincidence point.)

Therefore, if in the above inequality

$$
\begin{aligned}
& G\left(y_{n}, y_{n+1}, y_{n+1}, t\right) \geq \phi\left(G\left(y_{n}, y_{n+1}, y_{n+1}, t\right)\right)>G\left(y_{n}, y_{n+1}, y_{n+1}, t\right) \\
& H\left(y_{n}, y_{n+1}, y_{n+1}, t\right) \leq \psi\left(H\left(y_{n}, y_{n+1}, y_{n+1}, t\right)\right)<H\left(y_{n}, y_{n+1}, y_{n+1}, t\right) .
\end{aligned}
$$

By Lemma (2.5), which is a contradiction. Hence,

$$
\begin{gathered}
G\left(y_{n}, y_{n+1}, y_{n+1}, t\right) \geq \phi\left(G\left(y_{n-1}, y_{n}, y_{n}, t\right)\right) \\
H\left(y_{n}, y_{n+1}, y_{n+1}, t\right) \leq \psi\left(H\left(y_{n-1}, y_{n}, y_{n}, t\right)\right) .
\end{gathered}
$$

Thus, by Lemma (2.6), $\left\{y_{n}\right\}$ is a Cauchy sequence, which implies that $\left\{g x_{n}\right\}$ is a Cauchy sequence. Since $\mathrm{g}(X)$ is complete, so there exists $u \in g(X)$ such that

$$
\lim _{n \rightarrow \infty} y_{n}=\lim _{n \rightarrow \infty} f x_{n}=\lim _{n \rightarrow \infty} g x_{n}=u \text {. }
$$

Since $u \in g(X)$, so there exists $p \in X$ such that $g p=u$. Let $f p \neq u$. From (3.3.1)

$$
G\left(f x_{n}, f p, f p, t\right) \geq \phi\left(\min \left\{\mathrm{G}\left(g x_{n}, f x_{n}, f x_{n}, t\right), G(g p, f p, f p, t), G(g p, f p, f p, t)\right\}\right) .
$$

As $n \rightarrow \infty$, we get

$$
\begin{aligned}
G(u, f p, f p, t) & \geq \phi(\min \{G(u, u, u, t), G(u, f p, f p, t)\}) \\
\geq & \phi(\min \{1, G(u, f p, f p, t)\}) .
\end{aligned}
$$

Now, if $G(u, f p, f p, t) \geq \phi(1)=1$, this implies that 


$$
\begin{gathered}
G(u, f p, f p, t)=1, \\
H\left(f x_{n}, f p, f p, t\right) \leq \psi\left(\min \left\{G\left(g x_{n}, f x_{n}, f x_{n}, t\right), H(g p, f p, f p, t), H(g p, f p, f p, t)\right\}\right) .
\end{gathered}
$$

As $n \rightarrow \infty$, we get

$$
\begin{aligned}
H(u, f p, f p, t) & \leq \psi(\max \{H(u, u, u, t), H(u, f p, f p, t)\}) \\
& \leq \psi(\max \{1, H(u, f p, f p, t)\}) .
\end{aligned}
$$

Now, if $H(u, f p, f p, t) \leq \psi(0)=0$, this implies that $H(u, f p, f p, t)=0$, which is a contradiction, since $f p \neq u$. Hence

and

$$
G(u, f p, f p, t) \geq \phi(G(u, f p, f p, t))>G(u, f p, f p, t)
$$

$$
H(u, f p, f p, t) \leq \psi(H(u, f p, f p, t))<H(u, f p, f p, t),
$$

which is absurd. Hence, $f p=u$. Thus, $f p=g p=u$.

Hence, $p$ is a coincidence point of $f$ and $g$.

Now, we show that $p$ is unique. Assume that there exists another point $q$ in $X$ such that $f q=g q$. If $f p \neq f q$, then

$$
\begin{aligned}
G(f q, f p, f p, t) & \geq \phi(\min \{G(g q, f q, f q, t), G(g p, f p, f p, t), G(g p, f p, f p, t)\}) \\
& \geq \phi(\min \{G(f q, f q, f q, t), G(f p, f p, f p, t)\}) \geq \phi(1)=1 .
\end{aligned}
$$

This implies that

$$
G(f q, f p, f p, t)=1 .
$$

$H(f q, f p, f p, t) \leq \psi(\max \{H(g q, f q, f q, t), H(g p, f p, f p, t), H(g p, f p, f p, t)\})$

$$
\leq \psi(\max \{H(f q, f q, f q, t), H(f p, f p, f p, t)\}) \leq \psi(0)=0 .
$$

This implies that $H(f q, f p, f p, t)=0$.

By Lemma (2.5), which is a contradiction as $f p \neq \mathrm{fq}$.

Moreover, if $f$ and $g$ are semi-compatible, then from Proposition 2.10, $f$ and $g$ have a unique common fixed point. The proof using (3.3.2) is similar.

If we take $g=1$ in Theorem (3.3), we obtain the following result as a generalization of Theorem (3.3) of Mustafa et al. [13] to non-Archimedean generalized intuitionistic fuzzy metric spaces.

Corollary 3.4. Let $(X, \mathrm{G}, \mathrm{H}, *, \diamond)$ be a complete non-Archimedean generalized intuitionistic fuzzy metric space with $t * t \geq t$ and $(1-t) \diamond(1-t) \leq(1-t)$. If the mappings $f: X \rightarrow X$ satisfy for all $x, y, z \in X$ either

and

$$
G(f x, f y, f z, t) \geq \phi(\min \{G(x, f x, f x, t), G(y, f y, f y, t), G(z, f z, f z, t)\})
$$

$$
H(f x, f y, f z, t) \leq \psi(\max \{H(x, f x, f x, t), H(y, f y, f y, t), H(z, f z, f z, t)\}),
$$

or

and

$$
G(f x, f y, f z, t) \geq \phi(\min \{G(x, x, f x, t), G(y, y, f y, t), G(z, z, f z, t)\})
$$

$$
H(f x, f y, f z, t) \leq \psi(\max \{H(x, x, f x, t), H(y, y, f y, t), H(z, z, f z, t)\}),
$$

where $t>0$ and $\in \Phi, \psi \in \Psi$. Then $f$ has a unique fixed point. 
Example 3.5. Let $(X, \mathrm{G}, \mathrm{H}, *, \diamond)$ be a non-Archimedean generalized intuitionistic fuzzy metric space defined in the example. Define $f, g: X \rightarrow X$ as follows: $f x=x / 6$ and $g x=x / 3$ and define $\phi:[0,1] \rightarrow[0,1]$ as $\phi(\mathrm{t})=\sqrt{ } t$.

Then the hypotheses of Theorem (3.1) holds. Also $f$ and $g$ satisfy the condition (3.1.1) for all $x, y, z \in R$ and 0 is the unique common fixed point of $f$ and $g$.

\section{Properties $P$ and $Q$}

In this section, we shall show that maps satisfying the conditions of Theorems 3.1 and 3.3 and Corollary 3.4 possess properties $Q$ and $P$ respectively.

Theorem 4.1. Under the conditions of Theorem $3.1 f$ and $g$ have property $Q$.

Proof. From Theorem 3.1, $F(f) \cap F(g) \neq \phi$. Therefore, $F\left(f^{n}\right) \cap F\left(g^{n}\right) \neq \phi$ for each positive integer $n$. Let $n$ be a fixed positive integer greater than 1 and suppose that $U \in F\left(f^{n}\right) \cap F\left(g^{n}\right)$. We claim that $u \in F(f) \cap F(g)$.

Let $u \in F\left(f^{n}\right) \cap F\left(g^{n}\right)$. Then, for any positive integers $i, j, k, r, l, s$ satisfying $0 \leq i, j, r, k, l$, $s \leq n$, we have

$$
\begin{aligned}
G\left(f^{i} g^{j} u, f^{r} g^{l} u, f^{s} g^{k} u, t\right) & \geq \phi\left(G\left(g\left(f^{i-1} g^{j} u\right), g\left(f^{i-1} g^{l} u\right), g\left(f^{s-1} g^{k} u\right), t\right)\right) \\
& \geq \phi\left(G\left(f^{i-1} g^{j+1} u, f^{r-1} g^{l+1} u, f^{s-1} g^{k+1} u, t\right)\right) . \\
H\left(f^{i} g^{j} u, f^{r} g^{l} u, f^{s} g^{k} u, t\right) & \leq \psi\left(H\left(g\left(f^{i-1} g^{j} u\right), g\left(f^{i-1} g^{l} u\right), g\left(f^{s-1} g^{k} u\right), t\right)\right) \\
& \leq \psi\left(H\left(f^{i-1} g^{j+1} u, f^{r-1} g^{l+1} u, f^{s-1} g^{k+1} u, t\right)\right) .
\end{aligned}
$$

Define

$$
\begin{aligned}
& \delta=\min _{0 \leq i, r, j, l, s, k \leq n} G\left(f^{i} g^{j} u, f^{r} g^{l} u, f^{s} g^{k} u, t\right), \\
& \delta=\max _{0 \leq i, r, j, l, s, k \leq n} H\left(f^{i} g^{j} u, f^{r} g^{l} u, f^{s} g^{k} u, t\right),
\end{aligned}
$$

where $t>0$. Assume that $0 \leq \delta<1$, then it follows from (3.1.1) $\delta \geq \phi(\delta)>\delta, \delta \leq \psi(\delta)<\delta$, which is a contradiction and hence $\delta=0$.

In particular, $G(f u, u, u, t)=1$ and $G(g u, u, u, t)=1, H(f u, u, u, t)=1$ and $H(g u, u, u, t)=0$ for each $>0$ and hence $f u=g u=u$, implies that, $u \in F(f) \cap F(g)$.

Hence $f$ and $g$ have property $Q$.

Corollary 4.2. Under the conditions of Corollary 3.2, $f$ has property $P$.

Theorem 4.3. Under the conditions of Theorem 3.3, $f$ and $g$ have property $Q$.

Proof. From Theorem 3.3, $F(f) \cap F(g) \neq \varnothing$. Therefore, $F\left(f^{n}\right) \cap F\left(g^{n}\right) \neq \varnothing$ for each positive integer $n$. Let $n$ be a fixed positive integer greater than 1 and suppose that $U \in F\left(f^{n}\right) \cap F\left(g^{n}\right)$. We claim that $u \in F(f) \cap F(g)$. Let $u \in F\left(f^{n}\right) \cap F\left(g^{n}\right)$.

Then, for positive integers $i, j, r, l, s, k$ satisfying $0 \leq i, r, j, l, s, k \leq n$, we have 


$$
\begin{aligned}
& G\left(f^{i} g^{j} u, f^{r} g^{l} u, f^{s} g^{k} u, t\right) \geq \phi\left(\operatorname { m i n } \left\{G\left(g\left(f^{i-1} g^{j} u\right), f\left(f^{i-1} g^{j} u\right), f\left(f^{i-1} g^{j} u\right), t\right),\right.\right. \\
& \left.G\left(g\left(f^{r-1} g^{l} u\right), f\left(f^{r-1} g^{l} u\right), f^{r-1} g^{l} u\right), t\right), \\
& \left.G\left(g\left(f^{s-1} g^{k} u\right), f\left(f^{s-1} g^{k} u\right), f\left(f^{s-1} g^{k} u\right), t\right)\right\} \\
& \geq \phi\left(\operatorname { m i n } \left\{G\left(f^{i-1} g^{j+1} u, f^{i} g^{j} u, f^{i} g^{j} u, t\right),\right.\right. \\
& G\left(f^{r-1 g l+1} u, f^{r} g^{l} u, f^{r} g^{l} u, t\right), \\
& \left.G\left(f^{s-1} g^{k+1} u, f^{s} g^{k} u, f^{s} g^{k} u, t\right)\right\}, \\
& H\left(f^{i} g^{j} u, f^{r} g^{l} u, f^{s} g^{k} u, t\right) \leq \psi\left(\operatorname { m a x } \left\{H\left(g\left(f^{i-1} g^{j} u\right), f\left(f^{i-1} g^{j} u\right), f\left(f^{i-1} g^{j} u\right), t\right),\right.\right. \\
& H\left(g\left(f^{r-1} g^{l} u\right), f\left(f^{r-1} g^{l} u\right), f\left(f^{r l} g^{l} u\right), t\right), \\
& \left.H\left(g\left(f^{s-1} g^{k} u\right), f\left(f^{s-1} g^{k} u\right), f\left(f^{s-1} g^{k} u\right), t\right)\right\} \\
& \leq \psi\left(\operatorname { m a x } \left\{H\left(f^{i-1} g^{j+1} u, f^{i} g^{j} u, t\right), H\left(f^{r-1 g l+1} u, f^{r} g^{l} u, t\right)\right.\right. \text {, } \\
& \left.H\left(f^{s-1} g^{k+1} u, f^{s} g^{k} u, f^{s} g^{k} u, t\right)\right\} .
\end{aligned}
$$

Define

$$
\begin{gathered}
\delta=\min _{0 \leq i, r, j, l, s, k \leq n} G\left(f^{i} g^{j} u, f^{r} g^{l} u, f^{s} g^{k} u, t\right), \\
\delta=\max _{0 \leq i, r, j, l, s, k \leq n} H\left(f^{i} g^{j} u, f^{r} g^{l} u, f^{s} g^{k} u, t\right),
\end{gathered}
$$

where $t>0$.

Assume that $0 \leq \delta<1$, then it follows from (3.3.1)

$$
\begin{gathered}
\delta \geq \phi(\min \{\delta, \delta, \delta\})=\phi(\delta)>\delta, \\
\Delta \leq \psi(\max \{\delta, \delta, \delta\})=\psi(\delta)<\delta,
\end{gathered}
$$

which is a contradiction and hence $\delta=1$.

In particular, $G(f u, u, u, t)=1$ and $G(g u, u, u, t)=1, H(f u, u, u, t)=0$ and $H(g u, u, u, t)=0$ for each $t>0$ and hence $f u=g u=u$, implies that, $u \in F(f) \cap F(g)$. Hence $f$ and $g$ have property $Q$.

Corollory 4.4. Under the conditions of Corollary 3.4, $f$ has property $P$.

\section{References}

[1] Abbas, M. \& Rhoades, B. E. (2009). Common fixed point results for non-commuting mappings without continuity in generalized metric spaces, Applied Mathematics and Computation, 215, 262-269.

[2] Atanassov, K. (1983). Intuitionstic fuzzy sets, VII ITKR's session, Sofia (Deposed in central Science-Technical Library of Bulg. Academy of Science, 1697/84) (in Bulgarian)

[3] Dhage, B. C. (1992). Generalized metric spaces and mappings with fixed point, Bull. Calcutta Math. Soc., 84 (4), 329-336.

[4] George, A., \& Veeramani, P. (1994). On Some results in fuzzy metric spaces, Fuzzy Sets and Systems, 64, 395-399.

[5] Hu. X.-Q., \& Luo, Q. (2012). Coupled coincidence point theorem for contractions in generalized fuzzy metric spaces, Fixed Point Theory Appl, 2012, 196. 
[6] Kramosil, O., \& Michalek, J. (1975). Fuzzy metric and statistical metric spaces, Kybernetics, 11, 330-334.

[7] Mustafa, Z., \& Sims, B. (2006). A new approach to generalized metric space, J. Nonlinear Convex Analysis, 7, 289-297.

[8] Mustafa, Z., Obiedat, H., \& Awawdeh, F. (2008). Some fixed point theorem for mapping on Complete G-metric spaces, Fixed Point Theory Appl, 2008, Article ID 189870.

[9] Park, J. H. (2004). Intuitionistic fuzzy metric spaces, Chaos, Solitons, Fractals, 22, $1039-1046$.

[10] Rao, K. P. R., Altun, I., \& Bindu, S. H. (2011). Common Coupled fixed point theorem in generalized fuzzy metric spaces, Adv. Fuzzy Syst. 2011, Article ID 986748.

[11] Saadati, R., \& Park, J. H. (2006). On the intuitionistic fuzzy topological spaces, Chaos, Solitons, Fractals, 27, 331-344.

[12] Sun, G., \& Yang, K. (2010). Generalized fuzzy metric spaces with Properties, Res. J. Appl. Sci, 2, 673-678.

[13] Zadeh, L. A. (1965). Fuzzy sets, Inform. and Control, 8, 338-353. 